$\mathrm{J}$ o u r n a l of

Mathematics

and Applications

JMA No 41, pp 95-107 (2018)

\title{
On the Exponential Stability of a Neutral Differential Equation of First Order
}

\author{
Melek Gözen and Cemil Tunç
}

\begin{abstract}
In this work, we establish some assumptions that guaranteeing the global exponential stability (GES) of the zero solution of a neutral differential equation (NDE). We aim to extend and improves some results found in the literature.
\end{abstract}

AMS Subject Classification: 34K20, 93D09, 93D20.

Keywords and Phrases: (GES); (NDE); Time-varying delays.

\section{Introduction}

In [1], sufficient conditions for solutions of the (NDEs) form

$$
\frac{d}{d t}(x(t)+c(t) x(t-\tau))+p(t) x(t)+q(t) x(t-\sigma)=0
$$

to tend zero as $t \rightarrow \infty$ are established.

In $[4,8,10,15,17]$, it was considered a (NDE),

$$
\frac{d}{d t}(x(t)+p x(t-\tau))=-\alpha x(t)+b \tanh (x(t-\sigma))=0
$$

and the asymptotic stability (AS) of solutions are investigated.

In addition, some qualitative behaviors of solutions of equation (2) or some different models of that (NDE) were investigated in the relevant literature; for example, (S), (AS), (ES) in $[2,6,9,11,14,16,17-25]$, (GES) in [3], asymptotic behaviors in [13], oscillation and non-oscillation in $[5,7]$ and so on.

COPYRIGHT (c) by Publishing House of Rzeszów University of Technology P.O. Box 85, 35-959 Rzeszów, Poland 
In this paper, we deal with the following (NDE) with different variable delays:

$$
\frac{d}{d t}\left[x(t)+\sum_{i=1}^{2} p_{i}(t) x\left(t-\tau_{i}(t)\right)\right]+a(t) h(x(t))-\sum_{i=1}^{2} b_{i}(t) \tanh x\left(t-\sigma_{i}(t)\right)=0
$$

for $t \geq 0$ where $a_{i}, b_{i}:[0, \infty) \rightarrow[0, \infty)$ are continuously differentiable functions and $\sum_{i=1}^{2} a_{i}^{2}(t) \leq 1$. The functions $\tau_{i}():.[0, \infty) \rightarrow\left[0, \tau_{i}\right],\left(\tau_{i}>0\right)$ and $\sigma_{i}():.[0, \infty) \rightarrow$ $\left[0, \sigma_{i}\right],\left(\sigma_{i}>0\right)$ are bounded and continuously differentiable, and the functions $h$, $p_{1}$ and $p_{2}$ are continuous with $h(0)=0$. Let $r_{i}=\max \left\{\tau_{i}, \sigma_{i}\right\}>0,(i=1,2)$. Let $\mu_{1}, \mu_{2}, \mu_{3}, \mu_{4} \in(0,1)$ be positive constants such that $\tau_{1}^{\prime}(t) \leq \mu_{1}, \tau_{2}^{\prime}(t) \leq \mu_{2}$, $\sigma_{1}^{\prime}(t) \leq \mu_{3}$ and $\sigma_{2}^{\prime}(t) \leq \mu_{4}$. For each solution of (NDE) (3), we suppose that

$$
x_{0}(\theta)=\phi(\theta), \theta \in\left[-r_{i}, 0\right], \quad \text { where } \phi \in C\left(\left[-r_{i}, 0\right] ; R\right)
$$

\section{Stability Result}

Our stability result is given below.

Theorem. Let $K, \alpha_{0}, \alpha_{1}, \alpha_{2}, \alpha_{3}$ and $\alpha_{4}$ be positive constants. The zero solution of (NDE)(3) is global exponential stable if the following matrix inequalities hold:

$$
\begin{aligned}
& \Omega=\left[\begin{array}{cccccc}
\Omega_{11} & \Omega_{12} & \Omega_{13} & \Omega_{14} & \Omega_{15} & 0 \\
* & \Omega_{22} & \Omega_{23} & \Omega_{24} & \Omega_{25} & 0 \\
* & * & \Omega_{33} & \Omega_{34} & \Omega_{35} & 0 \\
* & * & * & \Omega_{44} & 0 & 0 \\
* & * & * & * & \Omega_{55} & 0 \\
* & * & * & * & * & \Omega_{66}
\end{array}\right]<0, \\
& \Delta=\left[\begin{array}{cccccc}
\Delta_{11} & \Delta_{12} & \Delta_{13} & \Delta_{14} & \Delta_{15} & 0 \\
* & \Delta_{22} & \Delta_{23} & \Delta_{24} & \Delta_{25} & 0 \\
* & * & \Delta_{33} & \Delta_{34} & \Delta_{35} & 0 \\
* & * & * & \Delta_{44} & 0 & 0 \\
* & * & * & * & \Delta_{55} & 0 \\
* & * & * & * & * & \Delta_{66}
\end{array}\right]<0
\end{aligned}
$$

where

$$
h_{1}(x)= \begin{cases}h(x) x^{-1}, & x \neq 0 \\ h^{\prime}(0), & x=0\end{cases}
$$

and

$$
\Omega_{11}=2 K \alpha_{0} \lambda_{1}-2 a(t) \alpha_{0} \frac{h(x)}{x} \lambda_{1}+\alpha_{1} \lambda_{1} \sum_{i=1}^{2} e^{2 K \tau_{i}}+\alpha_{3} \lambda_{1} \sum_{i=1}^{2} e^{2 K \sigma_{i}}
$$




$$
\begin{aligned}
& +\frac{\alpha_{2} \lambda_{1}}{2 K} \sum_{i=1}^{2}\left(e^{2 K \tau_{i}}-1\right)+\frac{\alpha_{4} \lambda_{1}}{2 K} \sum_{i=1}^{2}\left(e^{2 K \sigma_{i}}-1\right), \\
& \Omega_{12}=2 \lambda_{1} K \alpha_{0} p_{1}(t)-\lambda_{1} \alpha_{0} a(t) \frac{h(x)}{x} p_{1}(t), \\
& \Omega_{13}=2 \lambda_{1} K \alpha_{0} p_{2}(t)-\lambda_{1} \alpha_{0} a(t) \frac{h(x)}{x} p_{2}(t), \\
& \Omega_{14}=\lambda_{1} \alpha_{0} b_{1}(t), \\
& \Omega_{15}=\lambda_{1} \alpha_{0} b_{2}(t) \text {, } \\
& \Omega_{22}=2 \lambda_{1} K \alpha_{0} p_{1}^{2}(t)-\alpha_{1} \lambda_{1}\left(1-\mu_{1}\right), \\
& \Omega_{23}=2 \lambda_{1} K \alpha_{0} p_{1}(t) p_{2}(t) \text {, } \\
& \Omega_{24}=\lambda_{1} \alpha_{0} p_{1}(t) b_{1}(t) \\
& \Omega_{25}=\lambda_{1} \alpha_{0} p_{1}(t) b_{2}(t) \text {, } \\
& \Omega_{33}=2 \lambda_{1} K \alpha_{0} p_{2}^{2}(t)-\alpha_{1} \lambda_{1}\left(1-\mu_{2}\right) \text {, } \\
& \Omega_{34}=\lambda_{1} \alpha_{0} p_{2}(t) b_{1}(t) \\
& \Omega_{35}=\lambda_{1} \alpha_{0} p_{2}(t) b_{2}(t), \\
& \Omega_{44}=-\alpha_{3} \lambda_{1}\left(1-\mu_{3}\right), \\
& \Omega_{55}=-\alpha_{3} \lambda_{1}\left(1-\mu_{4}\right), \\
& \Omega_{66}=-\alpha_{2} \tau_{i} \text {, } \\
& \Delta_{11}=2 K \alpha_{0} \lambda_{2}-2 a(t) \alpha_{0} \frac{h(x)}{x} \lambda_{2}+\alpha_{1} \lambda_{2} \sum_{i=1}^{2} e^{2 K \tau_{i}}+\alpha_{3} \lambda_{2} \sum_{i=1}^{2} e^{2 K \sigma_{i}}, \\
& +\frac{\alpha_{2} \lambda_{2}}{2 K} \sum_{i=1}^{2}\left(e^{2 K \tau_{i}}-1\right)+\frac{\alpha_{4} \lambda_{2}}{2 K} \sum_{i=1}^{2}\left(e^{2 K \sigma_{i}}-1\right), \\
& \Delta_{12}=2 \lambda_{2} K \alpha_{0} p_{1}(t)-\lambda_{2} \alpha_{0} a(t) \frac{h(x)}{x} p_{1}(t), \\
& \Delta_{13}=2 \lambda_{2} K \alpha_{0} p_{2}(t)-\lambda_{2} \alpha_{0} a(t) \frac{h(x)}{x} p_{2}(t), \\
& \Delta_{14}=\lambda_{2} \alpha_{0} b_{1}(t) \text {, } \\
& \Delta_{15}=\lambda_{2} \alpha_{0} b_{2}(t) \text {, } \\
& \Delta_{22}=2 \lambda_{2} K \alpha_{0} p_{1}^{2}(t)-\alpha_{1} \lambda_{2}\left(1-\mu_{1}\right), \\
& \Delta_{23}=2 \lambda_{2} K \alpha_{0} p_{1}(t) p_{2}(t) \text {, } \\
& \Delta_{24}=\lambda_{2} \alpha_{0} p_{1}(t) b_{1}(t), \\
& \Delta_{25}=\lambda_{2} \alpha_{0} p_{1}(t) b_{2}(t), \\
& \Delta_{33}=2 \lambda_{2} K \alpha_{0} p_{2}^{2}(t)-\alpha_{1} \lambda_{1}\left(1-\mu_{2}\right), \\
& \Delta_{34}=\lambda_{2} \alpha_{0} p_{2}(t) b_{1}(t), \\
& \Delta_{35}=\lambda_{2} \alpha_{0} p_{2}(t) b_{2}(t), \\
& \Delta_{44}=-\alpha_{3} \lambda_{2}\left(1-\mu_{3}\right), \\
& \Delta_{55}=-\alpha_{3} \lambda_{2}\left(1-\mu_{4}\right), \\
& \Delta_{66}=-\alpha_{4} \sigma_{i}, \\
& \lambda_{1}=\frac{1}{2} \frac{\tau_{i}}{\tau_{i}+\sigma_{i}}, \quad \lambda_{2}=\frac{1}{2} \frac{\sigma_{i}}{\tau_{i}+\sigma_{i}}, \quad(i=1,2) .
\end{aligned}
$$


Proof. Choose an auxiliary functional, that is, Lyapunov functional (LF) by

$$
\begin{aligned}
V(.)=V(t, x)= & e^{2 K t} \alpha_{0}\left[x(t)+\sum_{i=1}^{2} p_{i}(t) x\left(t-\tau_{i}(t)\right)\right]^{2} \\
& +\alpha_{1} \sum_{i=1}^{2} \int_{t-\tau_{i}(t)}^{t} e^{2 K\left(s+\tau_{i}\right)} x^{2}(s) d s \\
& +\alpha_{2} \sum_{i=1}^{2} \int_{-\tau_{i}}^{0} \int_{t+\theta}^{t} e^{2 K(s-\theta)} x^{2}(s) d s d \theta \\
& +\alpha_{3} \sum_{i=1}^{2} \int_{t-\sigma_{i}(t)}^{t} e^{2 K\left(s+\sigma_{i}\right)} \tanh ^{2} x(s) d s \\
& +\alpha_{4} \sum_{i=1}^{2} \int_{-\sigma_{i}}^{0} \int_{t+\theta}^{t} e^{2 K(s-\theta)} \tanh ^{2} x(s) d s d \theta
\end{aligned}
$$

where $\alpha_{i} \in \Re,(i=0,1, \ldots, 4), \alpha_{i}>0$, and we choose them later.

The calculation of derivative of (LF) $V($.$) with respect to the (NDE) (3) gives that$

$$
\begin{aligned}
\frac{d V(.)}{d t}= & 2 K e^{2 K t} \alpha_{0}\left[x(t)+\sum_{i=1}^{2} p_{i}(t) x\left(t-\tau_{i}(t)\right)\right]^{2} \\
& +2 e^{2 K t} \alpha_{0}\left[x(t)+\sum_{i=1}^{2} p_{i}(t) x\left(t-\tau_{i}(t)\right)\right] \\
& \times\left[-a(t) h(x(t))+\sum_{i=1}^{2} b_{i}(t) \tanh x\left(t-\sigma_{i}(t)\right)\right] \\
& +\alpha_{1} \sum_{i=1}^{2} e^{2 K\left(t+\tau_{i}\right)} x^{2}(t)-\alpha_{1} \sum_{i=1}^{2}\left(1-\tau_{i}^{\prime}(t)\right) e^{2 K\left(t-\tau_{i}(t)+\tau_{i}\right)} x^{2}\left(t-\tau_{i}(t)\right) \\
& -\frac{\alpha_{2}}{2 K} \sum_{i=1}^{2}\left[e^{2 K t}-e^{2 K\left(t+\tau_{i}\right)}\right] x^{2}(t)-\alpha_{2} e^{2 K t} \sum_{i=1}^{2} \int_{t-\tau_{i}}^{t} x^{2}(s) d s \\
& +\alpha_{3} \sum_{i=1}^{2} e^{2 K\left(t+\sigma_{i}\right)} \tanh ^{2} x(t) \\
& -\alpha_{3} \sum_{i=1}^{2}\left(1-\sigma_{i}^{\prime}\right) e^{2 K\left(t-\sigma_{i}(t)+\sigma_{i}\right)} \tanh ^{2} x\left(t-\sigma_{i}(t)\right)
\end{aligned}
$$




$$
\begin{aligned}
& -\frac{\alpha_{4}}{2 K} \sum_{i=1}^{2}\left[e^{2 K t}-e^{2 K\left(t+\sigma_{i}\right)}\right] \tanh ^{2} x(t)-\alpha_{4} e^{2 K t} \sum_{i=1}^{2} \int_{t-\sigma_{i}}^{t} \tanh ^{2} x(s) d s \\
& =2 K e^{2 K t} \alpha_{0}\left[x^{2}(t)+2 x(t) p_{1}(t) x\left(t-\tau_{1}(t)\right)+2 x(t) p_{2}(t) x\left(t-\tau_{2}(t)\right)\right] \\
& +p_{1}^{2}(t) x^{2}\left(t-\tau_{1}(t)\right)+p_{2}^{2} x^{2}\left(t-\tau_{2}(t)\right) \\
& +2 p_{1}(t) p_{2}(t) x\left(t-\tau_{1}(t)\right) x\left(t-\tau_{2}(t)\right) \\
& +2 e^{2 K t} \alpha_{0}\left[-\alpha(t) \frac{h(x)}{x} x^{2}(t)+x(t) b_{1}(t) \tanh x\left(t-\sigma_{1}(t)\right)\right. \\
& +x(t) b_{2}(t) \tanh x\left(t-\sigma_{2}(t)\right)-a(t) \frac{h(x)}{x} x(t) p_{1}(t) x\left(t-\tau_{1}(t)\right) \\
& -a(t) \frac{h(x)}{x} x(t) p_{2}(t) x\left(t-\tau_{2}(t)\right) \\
& +\left(p_{1}(t) x\left(t-\tau_{1}(t)\right)+p_{2}(t) x\left(t-\tau_{2}(t)\right)\right)\left(b_{1}(t) \tanh x\left(t-\sigma_{1}(t)\right)\right. \\
& \left.\left.+b_{2}(t) \tanh x\left(t-\sigma_{2}(t)\right)\right)\right] \\
& +\alpha_{1} \sum_{i=1}^{2} e^{2 K\left(t+\tau_{i}\right)} x^{2}(t)-\alpha_{1}\left(1-\tau_{1}^{\prime}(t)\right) e^{2 K\left(t-\tau_{1}(t)+\tau_{1}\right)} x^{2}\left(t-\tau_{1}(t)\right) \\
& -\alpha_{1}\left(1-\tau_{2}^{\prime}(t)\right) e^{2 K\left(t-\tau_{2}(t)+\tau_{2}\right)} x^{2}\left(t-\tau_{2}(t)\right) \\
& +\frac{\alpha_{2}}{2 K} e^{2 K t} \sum_{i=1}^{2}\left[e^{2 K \tau_{i}}-1\right] x^{2}(t)-\alpha_{2} e^{2 K t} \sum_{i=1}^{2} \int_{t-\tau_{i}}^{t} x^{2}(s) d s \\
& +\alpha_{3} \sum_{i=1}^{2} e^{2 K\left(t+\sigma_{i}\right)} \tanh ^{2} x(t) \\
& -\alpha_{3}\left(1-\sigma_{1}^{\prime}(t)\right) e^{2 K\left(t-\sigma_{1}(t)+\sigma_{1}\right)} \tanh ^{2} x\left(t-\sigma_{1}(t)\right) \\
& -\alpha_{3}\left(1-\sigma_{2}^{\prime}(t)\right) e^{2 K\left(t-\sigma_{2}(t)+\sigma_{2}\right)} \tanh ^{2} x\left(t-\sigma_{2}(t)\right) \\
& +\frac{\alpha_{4}}{2 K} e^{2 K t} \sum_{i=1}^{2}\left[e^{2 K \sigma_{i}}-1\right] \tanh ^{2} x(t)-\alpha_{4} e^{2 K t} \sum_{i=1}^{2} \int_{t-\sigma_{i}}^{t} \tanh ^{2} x(s) d s .
\end{aligned}
$$

The assumptions of the theorem implies

$$
\begin{aligned}
& -\alpha_{1}\left(1-\tau_{1}^{\prime}(t)\right) e^{2 K\left(\tau_{1}-\tau_{1}(t)\right)} \leq-\alpha_{1}\left(1-\mu_{1}\right) \\
& -\alpha_{1}\left(1-\tau_{2}^{\prime}(t)\right) e^{2 K\left(\tau_{2}-\tau_{2}(t)\right)} \leq-\alpha_{1}\left(1-\mu_{2}\right) \\
& -\alpha_{3}\left(1-\sigma_{1}^{\prime}(t)\right) e^{2 K\left(\sigma_{1}-\sigma_{1}(t)\right)} \leq-\alpha_{3}\left(1-\mu_{3}\right)
\end{aligned}
$$

and

$$
-\alpha_{3}\left(1-\sigma_{2}^{\prime}(t)\right) e^{2 K\left(\sigma_{2}-\sigma_{2}(t)\right)} \leq-\alpha_{3}\left(1-\mu_{4}\right)
$$


Then,

$$
\begin{aligned}
& \frac{d V(.)}{d t} \leq 2 K e^{2 K t} \alpha_{0}\left[x^{2}(t)+2 x(t) p_{1}(t) x\left(t-\tau_{1}(t)\right)+2 x(t) p_{2}(t) x\left(t-\tau_{2}\right)\right) \\
& +p_{1}^{2}(t) x^{2}\left(t-\tau_{1}(t)\right)+p_{2}^{2}(t) x^{2}\left(t-\tau_{2}(t)\right) \\
& +2 p_{1}(t) p_{2}(t) x\left(t-\tau_{1}(t)\right) x\left(t-\tau_{2}(t)\right) \\
& +2 e^{2 K t} \alpha_{0}\left[-\alpha(t) \frac{h(x)}{x} x^{2}(t)+x(t) b_{1}(t) \tanh x\left(t-\sigma_{1}(t)\right)\right. \\
& +x(t) b_{2}(t) \tanh x\left(t-\sigma_{2}(t)\right)-a(t) \frac{h(x)}{x} x(t) p_{1}(t) x\left(t-\tau_{1}(t)\right) \\
& -a(t) \frac{h(x)}{x} x(t) p_{2}(t) x\left(t-\tau_{2}(t)\right) \\
& +p_{1}(t) b_{1}(t) x\left(t-\tau_{1}(t)\right) \tanh x\left(t-\sigma_{1}(t)\right) \\
& +p_{1}(t) b_{2}(t) x\left(t-\tau_{1}(t)\right) \tanh x\left(t-\sigma_{2}(t)\right) \\
& +p_{2}(t) b_{1}(t) x\left(t-\tau_{2}(t)\right) \tanh x\left(t-\sigma_{1}(t)\right) \\
& +p_{2}(t) b_{2}(t) x\left(t-\tau_{2}(t)\right) \tanh x\left(t-\sigma_{2}(t)\right) \\
& +\alpha_{1} \sum_{i=1}^{2} e^{2 K\left(t+\tau_{i}\right)} x^{2}(t)-\alpha_{1} e^{2 K t}\left(1-\mu_{1}\right) x^{2}\left(t-\tau_{1}(t)\right) \\
& -\alpha_{1} e^{2 K t}\left(1-\mu_{2}\right) x^{2}\left(t-\tau_{2}(t)\right) \\
& +\frac{\alpha_{2}}{2 K} e^{2 K t} \sum_{i=1}^{2}\left[e^{2 K \tau_{i}}-1\right] x^{2}(t)-\alpha_{2} e^{2 K t} \sum_{i=1}^{2} \int_{t-\tau_{i}}^{t} x^{2}(s) d s \\
& +\alpha_{3} \sum_{i=1}^{2} e^{2 K\left(t+\sigma_{i}\right)} \tanh ^{2} x(t)-\alpha_{3} e^{2 K t}\left(1-\mu_{3}\right) \tanh ^{2} x\left(t-\sigma_{1}(t)\right) \\
& -\alpha_{3} e^{2 K t}\left(1-\mu_{4}\right) \tanh ^{2} x\left(t-\sigma_{2}(t)\right) \\
& +\frac{\alpha_{4}}{2 K} e^{2 K t} \sum_{i=1}^{2}\left[e^{2 K \sigma_{i}}-1\right] \tanh ^{2} x(t) \\
& -\alpha_{4} e^{2 K t} \sum_{i=1}^{2} \int_{t-\sigma_{i}}^{t} \tanh ^{2} x(s) d s
\end{aligned}
$$

Since

$$
\tanh ^{2} x \leq x^{2}
$$

then

$$
\begin{aligned}
\frac{d V(.)}{d t} \leq & e^{2 K t}\left\{\left[2 K \alpha_{0}-2 \alpha_{0} a(t) \frac{h(x)}{x}+\alpha_{1} \sum_{i=1}^{2} e^{2 K \tau_{i}}+\frac{\alpha_{2}}{2 K} \sum_{i=1}^{2}\left(e^{2 K \tau_{i}}-1\right)\right.\right. \\
& \left.+\alpha_{3} \sum_{i=1}^{2} e^{2 K \sigma_{i}}+\frac{\alpha_{4}}{2 K} \sum_{i=1}^{2}\left(e^{2 K \sigma_{i}}-1\right)\right] x^{2}(t)
\end{aligned}
$$




$$
\begin{aligned}
& +\left[4 K \alpha_{0} p_{1}(t)-2 \alpha_{0} a(t) \frac{h(x)}{x} p_{1}(t)\right] x(t)\left(t-\tau_{1}(t)\right) \\
& +\left[4 K \alpha_{0} p_{2}(t)-2 \alpha_{0} a(t) \frac{h(x)}{x} p_{2}(t)\right] x(t)\left(t-\tau_{2}(t)\right) \\
& +2 \alpha_{0} b_{1}(t) x(t) \tanh x\left(t-\sigma_{1}(t)\right) \\
& +2 \alpha_{0} b_{2}(t) x(t) \tanh x\left(t-\sigma_{2}(t)\right) \\
& +\left[2 K \alpha_{0} p_{1}^{2}(t)-\alpha_{1}\left(1-\mu_{1}\right)\right] x^{2}\left(t-\tau_{1}(t)\right) \\
& +4 K \alpha_{0} p_{1}(t) p_{2}(t) x\left(t-\tau_{1}(t)\right) x\left(t-\tau_{2}(t)\right) \\
& +2 \alpha_{0} p_{1}(t) b_{1}(t) x\left(t-\tau_{1}(t)\right) \tanh x\left(t-\sigma_{1}(t)\right) \\
& +2 \alpha_{0} p_{1}(t) b_{2}(t) x\left(t-\tau_{1}(t)\right) \tanh x\left(t-\sigma_{2}(t)\right) \\
& +\left[2 K \alpha_{0} p_{2}^{2}(t)-\alpha_{1}\left(1-\mu_{2}\right)\right] x^{2}\left(t-\tau_{2}(t)\right) \\
& +2 \alpha_{0} p_{2}(t) b_{1}(t) x\left(t-\tau_{2}(t)\right) \tanh x\left(t-\sigma_{1}(t)\right) \\
& +2 \alpha_{0} p_{2}(t) b_{2}(t) x\left(t-\tau_{2}(t)\right) \tanh x\left(t-\sigma_{2}(t)\right) \\
& -\alpha_{3}\left(1-\mu_{3}\right) \tanh ^{2} x\left(t-\sigma_{1}(t)\right) \\
& -\alpha_{3}\left(1-\mu_{4}\right) \tanh ^{2} x\left(t-\sigma_{2}(t)\right) \\
& -\alpha_{2} \sum_{i=1}^{2} \int_{t-\tau_{i}}^{t} x^{2}(s) d s \\
& \left.-\alpha_{4} \sum_{i=1}^{t} \int_{t-\sigma_{i}}^{t} \tanh ^{2} x(s) d s\right\} .
\end{aligned}
$$

Then,

$$
\frac{d V(.)}{d t} \leq \sum_{i=1}^{2} \frac{1}{\tau_{i}} \int_{t-\tau_{i}}^{t} \xi_{1}^{T}(t, s) \Omega \xi_{1}(t, s) d s+\sum_{i=1}^{2} \frac{1}{\sigma_{i}} \int_{t-\sigma_{i}}^{t} \xi_{2}^{T}(t, s) \Delta \xi_{2}(t, s) d s,
$$

where

$$
\xi_{1}(t, s)=\left[x(t), x\left(t-\tau_{1}(t)\right), x\left(t-\tau_{2}(t)\right), \tanh x\left(t-\sigma_{1}(t)\right), \tanh x\left(t-\sigma_{2}(t)\right), x(s)\right]^{T}
$$

and

$\xi_{2}(t, s)=\left[x(t), x\left(t-\tau_{1}(t)\right), x\left(t-\tau_{2}(t)\right), \tanh x\left(t-\sigma_{1}(t)\right), \tanh x\left(t-\sigma_{2}(t)\right), \tanh x(s)\right]^{T}$.

From (4), we have $\frac{d V(.)}{d t}<0$, which implies that $V(.) \leq V(0, x(0))$. In view of the (LF) $V($.$) , we find$

$$
V(0, x(0))=\alpha_{0}\left[x(0)+\sum_{i=1}^{2} p_{i}(0) x\left(-\tau_{i}(0)\right)\right]^{2}+\alpha_{1} \sum_{i=1}^{2} \int_{-\tau_{i}(0)}^{0} e^{2 K\left(s+\tau_{i}\right)} x^{2}(s) d s
$$




$$
\begin{aligned}
& +\alpha_{2} \sum_{i=1}^{2} \int_{-\tau_{i}}^{0} \int_{\theta}^{0} e^{2 K(s-\theta)} x^{2}(s) d s d \theta+\alpha_{3} \sum_{i=1}^{2} \int_{-\sigma_{i}(0)}^{0} e^{2 K\left(s+\sigma_{i}\right)} \tanh ^{2} x(s) d s \\
& +\alpha_{4} \sum_{i=1}^{2} \int_{-\sigma_{i}}^{0} \int_{\theta}^{0} e^{2 K(s-\theta)} \tanh ^{2} x(s) d s d \theta .
\end{aligned}
$$

It is also obvious that

$$
\begin{aligned}
\alpha_{0}\left[x(0)+\sum_{i=1}^{2} p_{i}(0) x\left(-\tau_{i}(0)\right)\right]^{2}= & \alpha_{0}\left[x^{2}(0)+2 x(0) \sum_{i=1}^{2} p_{i}(0) x\left(-\tau_{i}(0)\right)\right. \\
& \left.+\left(\sum_{i=1}^{2} p_{i}(0) x\left(-\tau_{i}(0)\right)\right)^{2}\right] \\
= & \alpha_{0}\left[x^{2}(0)+2 x(0) \sum_{i=1}^{2} p_{i}(0) x\left(-\tau_{i}(0)\right)\right. \\
& \left.+p_{1}^{2}(0) x^{2}\left(-\tau_{1}(0)\right)\right) \\
& \left.+2 p_{1}(0) x\left(-\tau_{1}(0)\right)\right) p_{2}(0) x\left(-\tau_{2}(0)\right) \\
& \left.+p_{2}^{2}(0) x^{2}\left(-\tau_{2}(0)\right)\right] .
\end{aligned}
$$

If we use the inequality

$$
2|x y| \leq x^{2}+y^{2},
$$

then

$$
\begin{aligned}
\alpha_{0}\left[x(0)+\sum_{i=1}^{2} p_{i}(0) x\left(-\tau_{i}(0)\right)\right]^{2} \leq & \alpha_{0}\left[x^{2}(0)+2 x^{2}(0)+p_{1}^{2}(0) x^{2}\left(-\tau_{1}(0)\right)\right. \\
& +p_{2}^{2}(0) x^{2}\left(-\tau_{2}(0)\right)+p_{1}^{2}(0) x^{2}\left(-\tau_{1}(0)\right) \\
& +p_{1}^{2}(0) x^{2}\left(-\tau_{1}(0)+p_{2}^{2}(0) x^{2}\left(-\tau_{2}(0)\right)\right. \\
& \left.+p_{2}^{2}(0) x^{2}\left(-\tau_{2}(0)\right)\right] \\
= & \alpha_{0}\left[3 x^{2}(0)+3 p_{1}^{2}(0) x^{2}\left(-\tau_{1}(0)\right)\right. \\
& \left.\left.+3 p_{2}^{2}(0) x^{2}\left(-\tau_{2}(0)\right)\right)\right] .
\end{aligned}
$$

In view of the assumption $\sum_{i=1}^{2} p_{i}^{2}(t) \leq 1$, it follows that

$$
\begin{aligned}
\alpha_{0}\left[x(0)+\sum_{i=1}^{2} p_{i}(0) x\left(-\tau_{i}(0)\right)\right]^{2} & \left.\leq \alpha_{0}\left[3 x^{2}(0)+3 x^{2}\left(-\tau_{1}(0)\right)+3 x^{2}\left(-\tau_{2}(0)\right)\right)\right] \\
& \leq 9 \alpha_{0} \sup _{\theta \in\left[-r_{i}, 0\right]}|\phi(\theta)|^{2}, \\
\alpha_{1} \sum_{i=1}^{2} \int_{-\tau_{i}(0)}^{0} e^{2 K\left(s+\tau_{i}\right)} x^{2}(s) d s & \leq \alpha_{1} \sum_{i=1}^{2} e^{2 K \tau_{i}} \int_{-\tau_{i}(0)}^{0} \sup _{t \in\left[-\tau_{i}(0), 0\right]} e^{2 K t} x^{2}(t) d s
\end{aligned}
$$




$$
\begin{aligned}
& =\alpha_{1} \sum_{i=1}^{2} e^{2 K \tau_{i}} \sup _{t \in\left[-\tau_{i}(0), 0\right]} e^{2 K t} x^{2}(t) \tau_{i}(0) \\
& \leq \alpha_{1} \sum_{i=1}^{2} e^{2 K r_{i}} r_{i} \sup _{t \in\left[-\tau_{i}(0), 0\right]} e^{2 K t} x^{2}(t) \\
& \leq \alpha_{1} \sum_{i=1}^{2} e^{2 K r_{i}} r_{i} \sup _{\theta \in\left[-r_{i}, 0\right]}|\phi(\theta)|^{2}, \\
& \alpha_{2} \sum_{i=1}^{2} \int_{-\tau_{i}}^{0} \int_{\theta}^{0} e^{2 K(s-\theta)} x^{2}(s) d s d \theta \leq \alpha_{2} \sum_{i=1}^{2} \int_{-\tau_{i}}^{0}\left[\sup _{s \in[\theta, 0]} x^{2}(s) \int_{\theta}^{0} e^{2 K(s-\theta)} d s\right] d \theta \\
& =\alpha_{2} \sum_{i=1}^{2} \int_{-\tau_{i}}^{0} \sup _{s \in[-\theta, 0]} x^{2}(s)\left[\frac{1}{2 K} e^{-2 K \theta}-\frac{1}{2 K}\right] d \theta \\
& \leq \frac{1}{2 K} \alpha_{2} \sum_{i=1}^{2} \int_{-\tau_{i}}^{0} \sup _{s \in[-\theta, 0]} x^{2}(s) e^{-2 K \theta} d \theta \\
& \leq \alpha_{2} \sum_{i=1}^{2} \sup _{\theta \in\left[-r_{i}, 0\right]}|\phi(\theta)|^{2}\left[-\frac{1}{4 K^{2}}+\frac{1}{4 K^{2}} e^{2 K \tau_{i}}\right] \\
& \leq \frac{1}{4 K^{2}} \alpha_{2} \sum_{i=1}^{2} e^{2 K r_{i}} \sup _{\theta \in\left[-r_{i}, 0\right]}|\phi(\theta)|^{2}, \\
& \alpha_{3} \sum_{i=1}^{2} \int_{-\sigma_{i}(0)}^{0} e^{2 K\left(s+\sigma_{i}\right)} \tanh ^{2} x(s) d s \leq \alpha_{3} \sum_{i=1}^{2} e^{2 K \sigma_{i}} \int_{-\sigma_{i}(0)}^{0} e^{2 K s} x^{2}(s) d s \\
& \leq \alpha_{3} \sum_{i=1}^{2} e^{2 K \sigma_{i}} \int_{-\sigma_{i}(0)}^{0} \sup _{t \in\left[-\sigma_{i}(0), 0\right]} e^{2 K t} x^{2}(t) d s \\
& =\alpha_{3} \sum_{i=1}^{2} e^{2 K \sigma_{i}} \sup _{t \in\left[-\sigma_{i}(0), 0\right]} e^{2 K t} x^{2}(t) \sigma_{i}(0) \\
& \leq \alpha_{3} \sum_{i=1}^{2} e^{2 K r_{i}} r_{i} \sup _{t \in\left[-\sigma_{i}(0), 0\right]} e^{2 K t} x^{2}(t), \\
& \alpha_{4} \sum_{i=1}^{2} \int_{-\sigma_{i}}^{0} \int_{\theta}^{0} e^{2 K(s-\theta)} \tanh ^{2} x(s) d s d \theta \leq \alpha_{4} \sum_{i=1}^{2} \int_{-\sigma_{i}}^{0}\left[\sup _{s \in[\theta, 0]} x^{2}(s) \int_{\theta}^{0} e^{2 K(s-\theta)} d s\right] d \theta \\
& =\alpha_{4} \sum_{i=1}^{2} \int_{-\sigma_{i}}^{0} \sup _{s \in[-\theta, 0]} x^{2}(s)\left[\frac{1}{2 K} e^{-2 K \theta}-\frac{1}{2 K}\right] d \theta \\
& \leq \frac{1}{2 K} \alpha_{4} \sum_{i=1}^{2} \int_{-\sigma_{i}}^{0} \sup _{s \in[-\theta, 0]} x^{2}(s) e^{-2 K \theta} d \theta
\end{aligned}
$$




$$
\begin{aligned}
& \leq \alpha_{4} \sum_{i=1}^{2} \sup _{\theta \in\left[-r_{i}, 0\right]}|\phi(\theta)|^{2}\left[-\frac{1}{4 K^{2}}+\frac{1}{4 K^{2}} e^{2 K \sigma_{i}}\right] \\
& \leq \frac{1}{4 K^{2}} \alpha_{4} \sum_{i=1}^{2} e^{2 K r_{i}} \sup _{\theta \in\left[-r_{i}, 0\right]}|\varphi(\theta)|^{2} .
\end{aligned}
$$

Hence,

$$
\begin{aligned}
V(0, x(0))= & \alpha_{0}\left[x(0)+\sum_{i=1}^{2} p_{i}(0) x\left(-\tau_{i}(0)\right)\right]^{2}+\alpha_{1} \sum_{i=1}^{2} \int_{-\tau_{i}(0)}^{0} e^{2 K\left(s+\tau_{i}\right)} x^{2}(s) d s \\
& +\alpha_{2} \sum_{i=1}^{2} \int_{-\tau_{i}}^{0} \int_{\theta}^{0} e^{2 K(s-\theta)} x^{2}(s) d s d \theta+\alpha_{3} \sum_{i=1}^{2} \int_{-\sigma_{i}(0)}^{0} e^{2 K\left(s+\sigma_{i}\right)} \tanh ^{2} x(s) d s \\
& +\alpha_{4} \sum_{i=1}^{2} \int_{-\sigma_{i}}^{0} \int_{\theta}^{0} e^{2 K(s-\theta)} \tanh ^{2} x(s) d s d \theta \\
\leq & {\left[9 \alpha_{0}+\left(\alpha_{1}+\alpha_{3}\right) \sum_{i=1}^{2} r_{i} e^{2 K r_{i}}+\left(\alpha_{2}+\alpha_{4}\right) \frac{1}{4 K^{2}} \sum_{i=1}^{2} e^{2 K r_{i}}\right] \sum_{i=1}^{2} \sup _{\theta \in\left[r_{i}, 0\right]}|\phi(\theta)|^{2} } \\
\equiv & M .
\end{aligned}
$$

We can now write

$$
\left|x+\sum_{i=1}^{2} p_{i}(t) x\left(-\tau_{i}(t)\right)\right|^{2} \leq M_{1} e^{-2 k t},
$$

where $M_{1}=\frac{M}{\alpha_{0}}>0$. For $\forall \varepsilon \in\left(0, \min \left\{2 K,-\frac{2}{r_{i}} \log \left|p_{i}(t)\right|\right\}\right)$ and $v>0$, the inequality $x y \leq v x^{2}+\frac{1}{v} y^{2}$ for any $x, y \in R$ implies that

$$
\begin{aligned}
e^{\varepsilon t}|x|^{2} & \leq(1+v) e^{\varepsilon t}\left|x(t)+\sum_{i=1}^{2} p_{i}(t) x\left(t-\tau_{i}(t)\right)\right|^{2}+\frac{1+v}{v} e^{\varepsilon t} \sum_{i=1}^{2}\left|p_{i}(t) x\left(t-\tau_{i}(t)\right)\right|^{2} \\
& \leq(1+v) M_{1}+\frac{1+v}{v} \sum_{i=1}^{2}\left|p_{i}(t)\right|^{2}\left|x\left(t-\tau_{i}(t)\right)\right|^{2} e^{\varepsilon r_{i}} e^{\varepsilon\left(t-\tau_{i}(t)\right)} .
\end{aligned}
$$

And from $\forall \varepsilon \in\left(0, \min \left\{2 K,-\frac{2}{r_{i}} \log \left|p_{i}(t)\right|\right\}\right)$, we have $\sum_{i=1}^{2}\left|p_{i}(t)\right|^{2} e^{\varepsilon r_{i}}<1$. Thus, if we choose $v>0$ sufficiently large, then it follows that

$$
\gamma=\frac{\sum_{i=1}^{2}\left|p_{i}(t)\right|^{2}(1+v) e^{\varepsilon r_{i}}}{v}<1 .
$$


Therefore,

$$
\begin{array}{r}
e^{\varepsilon t}|x|^{2} \leq(1+v) M_{1}+\gamma \sum_{i=1}^{2}\left|x\left(t-\tau_{i}(t)\right)\right|^{2} e^{\varepsilon\left(t-\tau_{i}(t)\right)} \quad(\forall T \geq 0), \\
\sup _{0 \leq t \leq T}\left\{e^{\varepsilon t}|x(t)|^{2}\right\} \leq(1+v) M_{1}+\gamma \sup _{\theta \in\left[r_{i}, 0\right]}|\varphi(\theta)|^{2}+\gamma \sup _{0 \leq t \leq T}\left\{e^{\varepsilon t}|x(t)|^{2}\right\}, \quad(i=1,2) .
\end{array}
$$

Consequently, we obtain

$$
\sup _{0 \leq t \leq T}\left\{e^{\varepsilon t}|x|^{2}\right\} \leq \frac{(1+v) M_{1}+\gamma \sup _{\theta \in\left[r_{i}, 0\right]}|\varphi(\theta)|^{2}}{1-\gamma}, \quad(i=1,2) .
$$

When $T \rightarrow+\infty$, we can find that

$$
\sup _{0 \leq t \leq \infty}\left\{e^{\varepsilon t}|x(t)|^{2}\right\} \leq \frac{(1+v) M_{1}+\gamma \sup _{\theta \in\left[r_{i}, 0\right]}|\varphi(\theta)|^{2}}{1-\gamma}, \quad(i=1,2) .
$$

Thus,

$$
|x| \leq M_{2} e^{-a t}
$$

where

$$
M_{2}=\sqrt{\frac{(1+v) M_{1}+\gamma \sup _{\theta \in\left[r_{i}, 0\right]}|\varphi(\theta)|^{2}}{1-\gamma}}>0, \alpha=\frac{\varepsilon}{2}>0, \quad(i=1,2) .
$$

This ends the proof.

\section{References}

[1] R.P. Agarwal, S.R. Grace, Asymptotic stability of certain neutral differential equations, Math. Comput. Modelling 31 (8-9) (2000) 9-15.

[2] H. Chen, X. Meng, An improved exponential stability criterion for a class of neutral delayed differential equations, Appl. Math. Lett. 24 (11) (2011) 1763-1767.

[3] H. Chen, Some improved criteria on exponential stability of neutral differential equation, Adv. Difference Equ. 2012 (2012:170) 9 pp.

[4] S. Deng, X. Liao, S. Guo, Asymptotic stability analysis of certain neutral differential equations: a descriptor system approach, Math. Comput. Simulation 79 (10) (2009) 2981-2993.

[5] H.A. El-Morshedy, K. Gopalsamy, Non-oscillation, oscillation and convergence of a class of neutral equations. Lakshmikantham's legacy: a tribute on his 75th birthday, Nonlinear Anal. 40 (1-8) (2000) Ser. A: Theory Methods 173-183. 
[6] E. Fridman, New Lyapunov-Krasovskii functionals for stability of linear retarded and neutral type systems, Systems Control Lett. 43 (4) (2001) 309-319.

[7] M.R.S. Kulenovic, G. Ladas, A. Meimaridou, Necessary and sufficient condition for oscillations of neutral differential equations, J. Austral. Math. Soc. Ser. B 28 (3) (1987) 362-375.

[8] O.M. Kwon, Ju H. Park, On improved delay-dependent stability criterion of certain neutral differential equations, Appl. Math. Comput. 199 (1) (2008) 385-391.

[9] X. Li, Global exponential stability for a class of neural networks, Appl. Math. Lett. 22 (8) (2009) 1235-1239.

[10] P.T. Nam, V.N. Phat, An improved stability criterion for a class of neutral differential equations, Appl. Math. Lett. 22 (1) (2009) 31-35.

[11] Ju H. Park, S. Won, Stability analysis for neutral delay-differential systems, J. Franklin Inst. 337 (1) (2000) 1-9.

[12] Ju H. Park, Ho Y. Jung, On the exponential stability of a class of nonlinear systems including delayed perturbations, J. Comput. Appl. Math. 159 (2) (2003) 467-471.

[13] J.H. Park, Delay-dependent criterion for asymptotic stability of a class of neutral equations, Appl. Math. Lett. 17 (10) (2004) 1203-1206.

[14] Ju H. Park, O. Kwon, On new stability criterion for delay-differential systems of neutral type, Appl. Math. Comput. 162 (2) (2005) 627-637.

[15] Ju H. Park, O.M. Kwon, Stability analysis of certain nonlinear differential equation, Chaos Solitons Fractals 37 (2) (2008) 450-453.

[16] T. Rojsiraphisal, P. Niamsup, Exponential stability of certain neutral differential equations, Appl. Math. Comput. 217 (8) (2010) 3875-3880.

[17] Y.G. Sun, L. Wang, Note on asymptotic stability of a class of neutral differential equations, Appl. Math. Lett. 19 (9) (2006) 949-953.

[18] C. Tunç, Asymptotic stability of nonlinear neutral differential equations with constant delays: a descriptor system approach, Ann. Differential Equations 27 (1) (2011) 1-8.

[19] C. Tunç, Exponential stability to a neutral differential equation of first order with delay, Ann. Differential Equations 29 (3) (2013) 253-256.

[20] C. Tunç, Asymptotic stability of solutions of a class of neutral differential equations with multiple deviating arguments, Bull. Math. Soc. Sci. Math. Roumanie (N.S.) 57 (105) (1) (2014) 121-130.

[21] C. Tunç, On the uniform asymptotic stability to certain first order neutral differential equations, Cubo 16 (2) (2014) 111-119. 
On the Exponential Stability of a Neutral Differential Equation of First Order 107

[22] C. Tunç, Convergence of solutions of nonlinear neutral differential equations with multiple delays, Bol. Soc. Mat. Mex. 21 (2) (2015) 219-231.

[23] C. Tunç, Y. Altun, Asymptotic stability in neutral differential equations with multiple delays, J. Math. Anal. 7 (5) (2016) 40-53.

[24] C. Tunç, A. Sirma, Stability analysis of a class of generalized neutral equations, J. Comput. Anal. Appl. 12 (4) (2010) 754-759.

[25] S. Xu, J. Lam, Improved delay-dependent stability criteria for time-delay systems, IEEE Trans. Automat. Control 50 (3) (2005) 384-387.

\section{DOI: $10.7862 /$ rf.2018.8}

\section{Melek Gözen}

email: melekgozen2013@gmail.com

ORCID: 0000-0002-7487-9869

Department of Business Administration

Management Faculty

Van Yuzuncu Yil University 65080, Erciş

TURKEY

\section{Cemil Tunç}

email: cemtunc@yahoo.com

ORCID: 0000-0003-2909-8753

Department of Mathematics

Faculty of Sciences

Van Yuzuncu Yll University

65080, Van

TURKEY

Received 0\%.09.2017

Accepted 13.04.2018 